\title{
GENERALIZED SUPPLEMENTED LATTICES
}

\author{
ÇIĞDEM BIÇER, CELIL NEBIYEV, AND ALI PANCAR
}

Received 19 April, 2016

\begin{abstract}
In this work, we define (amply) generalized supplemented lattices and investigate some properties of these lattices. In this paper, all lattices are complete modular lattices with the smallest element 0 and the greatest element 1. Let $L$ be a lattice, $1=a_{1} \vee a_{2} \vee \ldots \vee a_{n}$ and the quotient sublattices $a_{1} / 0, a_{2} / 0, \ldots, a_{n} / 0$ be generalized supplemented, then $L$ is generalized supplemented. If $L$ is an amply generalized supplemented lattice, then for every $a \in L$, the quotient sublattice $1 / a$ is amply generalized supplemented.
\end{abstract}

2010 Mathematics Subject Classification: 06C05; 06C15

Keywords: lattices, supplemented lattices, amply supplemented lattices, hollow lattices

\section{INTRODUCTION}

Throughout this paper, all lattices are complete modular lattices with the smallest element 0 and the greatest element 1 . Let $L$ be a lattice, $a, b \in L$ and $a \leq b$. A sublattice $\{x \in L \mid a \leq x \leq b\}$ is called a quotient sublattice, denoted by $b / a$. An element $a^{\prime}$ of a lattice $L$ is called a complement of $a$ if $a \wedge a^{\prime}=0$ and $a \vee a^{\prime}=1$. A lattice $L$ is said to be complemented if each element in $L$ has at least one complement. An element $c$ of $L$ is said to be compact if for every subset $X$ of $L$ such that $c \leq \vee X$ there is a finite $F \subseteq X$ such that $c \leq \vee F$. A lattice $L$ is said to be compactly generated if each of its elements is a join of compact elements. An element $a$ of $L$ is said to be small or superfluous if $a \vee b \neq 1$ holds for every $b \neq 1$ and denoted by $a \ll L$. The meet of all the maximal elements $(\neq 1)$ of a lattice $L$ is called the radical of $L$ and denoted by $r(L)$. An element $c$ of $L$ is called a supplement of $b$ in $L$ if it is minimal for $b \vee c=1$. $a$ is a supplement of $b$ in lattice $L$ if and only if $a \vee b=1$ and $a \wedge b \ll a / 0 . L$ is called a supplemented lattice if every element of $L$ has a supplement in $L$. We say that an element $b$ of $L$ lies above an element $a$ of $L$ if $a \leq b$ and $b \ll 1 / a$. $L$ is said to be hollow if every element $(\neq 1)$ is superfluous in $L$, and $L$ is said to be local if $L$ has a greatest element $(\neq 1)$. An element $a$ of $L$ is called a weak supplement of $b$ in $L$ if $a \vee b=1$ and $a \wedge b \ll L . L$ is called a weakly supplemented lattice, if every element of $L$ has a weak supplement in $L$. An element $a \in L$ has ample supplements in $L$ if for every $b \in L$ with $a \vee b=1, a$ has a supplement $b^{\prime}$ in $L$ with $b^{\prime} \leq b$. L is called an amply supplemented lattice, if every 
element of $L$ has ample supplements in $L$. It is clear that every supplemented lattice is weakly supplemented and every amply supplemented lattice is supplemented.

More information about (amply) supplemented lattices are in [1,2] and [3]. More results about (amply) supplemented modules are in [4,6] and [7]. The definitions of (amply) generalized supplemented modules and some properties of them are in [5]. We generalize some properties of (amply) generalized supplemented modules.

In this paper, we constitute relationships between generalized supplemented quotient sublattices and generalized supplemented lattices by Corollary 1, Theorem 3 and Corollary 2. We also constitute relationships between amply generalized supplemented quotient sublattices and amply generalized supplemented lattices by Proposition 3 and Proposition 5.

\section{GENERALIZED SUPPLEMENTED LATTICES}

In this part, generalized supplement elements and generalized supplemented lattices are defined and some properties of them are given.

Definition 1. Let $L$ be a lattice and $a \in L$. An element $b \in L$ is called a generalized supplement or Rad-supplement of $a$ in $L$ if $a \vee b=1$ and $a \wedge b \leq r(b / 0)$. In this fact, $a$ has a generalized supplement (or Rad-supplement) in $L$. $L$ is said to be generalized supplemented or Rad-supplemented if every element of $L$ has a generalized supplement or Rad-supplement in $L$.

It is clear that every supplement element is generalized supplement in a lattice, also supplemented lattices are generalized supplemented but a generalized supplemented lattice need not to be supplemented. We give an example about this in the next part.

Lemma 1 ([3], Theorem 1.5). Let $L$ be a lattice and $a, b \in L$. Then the quotient sublattices $(a \vee b) / b$ and $a /(a \wedge b)$ are isomorphic.

Lemma 2. Let $L$ be a lattice and $c$ be a generalized supplement of $b$ in $L$.

(a) If $a \leq b$ and $a \vee c=1$ then $c$ is a generalized supplement of $a$ in $L$.

(b) $r(c / 0)=c \wedge r(L)$.

Proof. (a) Since $c$ is a generalized supplement of $b$ in $L, b \vee c=1$ and $b \wedge c \leq$ $r(c / 0)$. Since $a \leq b, a \wedge c \leq b \wedge c \leq r(c / 0)$. Thus $c$ is a generalized supplement of $a$ in $L$.

(b) $r(c / 0) \leq c \wedge r(L)$ is clear. Let $x$ be a maximal element $(\neq c)$ in $c / 0$. Since $\frac{1}{b \vee x}=\frac{c \vee b}{b \vee x}=\frac{c \vee b \vee x}{b \vee x} \cong \frac{c}{c \wedge(b \vee x)}=\frac{c}{(c \wedge b) \vee x}=\frac{c}{x}, b \vee x$ is a maximal element $(\neq 1)$ of $L$. Hence $r(L) \leq b \vee x$ and $c \wedge r(L) \leq c \wedge(b \vee x)=(c \wedge b) \vee x=x$. Since $c \wedge$ $r(L) \leq x$ for every maximal element $(\neq c)$ in $c / 0, c \wedge r(L) \leq r(c / 0)$ and $r(c / 0)=$ $c \wedge r(L)$.

Theorem 1. Let $L$ be a lattice and $b$ be a maximal element $(\neq 1)$ in $L$. If $c$ is a generalized supplement of $b$ in $L$ then $b \wedge c=r(c / 0)$ and $r(c / 0)$ is the unique maximal element $(\neq c)$ of $c / 0$. 
Proof. Since $b$ is maximal $(\neq 1)$ in $L$, by Lemma $1, b \wedge c$ is a maximal element $(\neq c)$ in $c / 0$. Because of definition of the radical, $r(c / 0) \leq b \wedge c$, and $c$ being generalized supplement of $b$ in $L, b \wedge c \leq r(c / 0)$. Hence $b \wedge c=r(c / 0)$ is the unique maximal element in $c / 0$.

Theorem 2. Let $L$ be a lattice and $1 \neq b \in L$. If $c$ is a generalized supplement of $b$ in $L$ and $r(L) \ll L$, then there exists a maximal element $m(\neq 1)$ in $L$ such that $b \leq m$.

Proof. If $b \leq r(L)$, then it is clear. Let $b \not \leq r(L)$. Then $r(c / 0)=c \wedge r(L) \neq c$ by Lemma $2(b)$. Hence there exists an element $x \leq c$ such that $x$ is a maximal element $(\neq c)$ in $c / 0$. Let $m=b \vee x$. By Lemma 1 and modularity $\frac{1}{m}=\frac{1}{b \vee x}=$ $\frac{b \vee c}{b \vee x} \cong \frac{c}{c \wedge(b \vee x)}=\frac{c}{(c \wedge b) \vee x}=\frac{c}{x}$ and so $m$ is a maximal element $(\neq 1)$ in $L$ such that $b \leq m$.

Lemma 3 ([3], Lemma 7.8 (i)). Let $L$ be a lattice. Then $a \vee r(L) \leq r(1 / a)$ for every $a \in L$.

Lemma 4 ([3], Exercise 7.3). Let $L$ be a lattice and $a \in L$. Then $r(a / 0) \leq r(L)$.

Lemma 5. Let $L$ be a lattice and $c$ be a generalized supplement of $b$ in $L$. Then for $a \leq b, a \vee c$ is a generalized supplement of $b$ in $1 / a$.

Proof. Since $c$ is a generalized supplement of $b$ in $L, b \vee c=1$ and $b \wedge c \leq r(c / 0)$. Then $b \vee(a \vee c)=1$ and by Lemma 3 and Lemma $4,(a \vee c) \wedge b=a \vee(b \wedge c) \leq a \vee$ $r(c / 0) \leq a \vee r((a \vee c) / 0) \leq r((a \vee c) / a)$. Hence $a \vee c$ is a generalized supplement of $b$ in $1 / a$.

Corollary 1. Let $L$ be a lattice. If $L$ is generalized supplemented and $a \in L$, then $1 / a$ is also generalized supplemented.

Proof. Clear from Lemma 5.

Lemma 6 ([3], Lemma 12.3). Let $L$ be a lattice. Then for every $a, b, c \in L$, $[(b \vee c) \wedge a] \leq[b \wedge(a \vee c)] \vee[c \wedge(a \vee b)]$.

Lemma 7. Let $L$ be a lattice and $a, b, c \in L$. If $a / 0$ is generalized supplemented and $a \vee b$ has a generalized supplement in $L$, then $b$ has a generalized supplement in $L$.

Proof. Let $c$ be a generalized supplement of $a \vee b$ in $L$ and $d$ be a generalized supplement of $(b \vee c) \wedge a$ in $a / 0$. Clearly, $1=a \vee b \vee c=b \vee c \vee d,(b \vee c) \wedge d \leq$ $r(d / 0), b \wedge(c \vee d) \leq[c \wedge(b \vee d)] \vee[d \wedge(b \vee c)] \leq r(c / 0) \vee r(d / 0) \leq r((c \vee d) / 0)$. Hence $c \vee d$ is a generalized supplement of $b$ in $L$.

Theorem 3. Let $L$ be a lattice $a, b \in L$ and $a \vee b=1$. If $a / 0$ and $b / 0$ are generalized supplemented, then $L$ is generalized supplemented. 
Proof. Let $c$ be arbitrary in L. $a \vee(b \vee c)=1$ has a generalized supplement 0 in $L$. Since $a / 0$ is generalized supplemented, by Lemma $7, b \vee c$ has a generalized supplement in $L$. And since $b / 0$ is generalized supplemented, $c$ has a generalized supplement in $L$. So, $L$ is generalized supplemented.

Corollary 2. If $1=a_{1} \vee a_{2} \vee \ldots \vee a_{n}$ and the quotient sublattices $a_{1} / 0, a_{2} / 0, \ldots$, $a_{n} / 0$ be generalized supplemented, then $L$ is generalized supplemented.

If $a<b$ and $a \leq c<b$ implies that $c=a$, then $a$ is said to be covered by $b$. If 0 is covered by $a$ for some element $a$ of $L$, then $a$ is called an atom. A lattice $L$ is said to be semiatomic if 1 is a join of atoms in L. [3]

Lemma 8 ([3], Theorem 6.7). Every modular compactly generated complemented lattice is semiatomic.

Proposition 1. Let $L$ be a modular compactly generated lattice. If $L$ is generalized supplemented, then the quotient sublattice $1 / r(L)$ is semiatomic.

Proof. Clearly, $1 / r(L)$ is a modular compactly generated lattice. Let $a \in 1 / r(L)$. Since $L$ is generalized supplemented, $a$ has a generalized supplement $b$ in $L$. Then we have $a \vee(b \vee r(L))=1$ and $a \wedge(b \vee r(L))=r(L)$. Hence $1 / r(L)$ is complemented and by Lemma 8,1/r(L) is semiatomic.

Proposition 2. Let $L$ be a lattice. If $L$ is generalized supplemented and $r(L) \ll L$, then $L$ is weakly supplemented.

Proof. Clear.

\section{AMPLY GENERALIZED SUPPLEMENTED LATTICES}

In this section, we define amply generalized supplemented or amply Rad-supplemented lattices and we give some properties of them.

Definition 2. Let $L$ be a lattice. We say an element $a$ of $L$ has ample generalized supplements in $L$ if for every $b$ of $L$ with $a \vee b=1, a$ has a generalized supplement $b^{\prime}$ in $L$ with $b^{\prime} \leq b . \quad L$ is called amply generalized supplemented or amply Radsupplemented if every element of $L$ has ample generalized supplements in $L$.

It is clear that every amply generalized supplemented lattice is generalized supplemented. But the converse is not always true (see Example 1). Every amply supplemented modular lattice is amply generalized supplemented.

Proposition 3. Let $L$ be a lattice. If $L$ is amply generalized supplemented, then the quotient sublattice 1/a is also amply generalized supplemented for every element a of $L$.

Proof. Let $a \in L$ and $x, y \in 1 / a$ with $x \vee y=1$. Since $L$ is amply generalized supplemented, there exists an element $y^{\prime}$ of $L$ such that $y^{\prime} \leq y, x \vee y^{\prime}=1$ and $x \wedge$ 
$y^{\prime} \leq r\left(y^{\prime} / 0\right)$. Then $x \wedge\left(a \vee y^{\prime}\right)=a \vee\left(x \wedge y^{\prime}\right) \leq a \vee r\left(y^{\prime} / 0\right) \leq r\left(\left(a \vee y^{\prime}\right) / a\right)$. Hence $a \vee y^{\prime}$ is a generalized supplement of $x$ in $1 / a$ with $a \vee y^{\prime} \leq y$.

Proposition 4. Let $L$ be a lattice, $a, b \in L$ and $a \vee b=1$. If $a$ and $b$ have ample generalized supplements in $L$, then $a \wedge b$ has ample generalized supplements in $L$.

Proof. Let $(a \wedge b) \vee c=1$ with $c \in L$. We clearly see that $b \vee(a \wedge c)=1$ and $a \vee(b \wedge c)=1$. Since $a \vee(b \wedge c)=1$ and $a$ has ample generalized supplements in $L$, then there exists an element $x$ of $L$ such that $x \leq b \wedge c, a \vee x=1$ and $a \wedge x \leq r(x / 0)$. Since $b \vee(a \wedge c)=1$ and $b$ has ample generalized supplements in $L$, then there exists an element $y$ of $L$ such that $y \leq a \wedge c, b \vee y=1$ and $b \wedge y \leq r(y / 0)$. Since $x \leq b$ and $a \vee x=1, b=b \wedge 1=b \wedge(a \vee x)=(a \wedge b) \vee x$. Similarly, we see that $a=(a \wedge b) \vee y$. Then $1=a \vee b=[(a \wedge b) \vee y] \vee[(a \wedge b) \vee x]=(a \wedge b) \vee x \vee y$. Since $a \wedge x \leq r(x / 0)$ and $b \wedge y \leq r(y / 0)$, then $(a \wedge b) \wedge(x \vee y)=(a \wedge x) \vee(b \wedge y) \leq$ $r(x / 0) \vee r(y / 0) \leq r((x \vee y) / 0)$. Hence $x \vee y$ is a generalized supplement of $a \wedge b$ in $L$ with $x \vee y \leq c$.

Proposition 5. Let $L$ be a lattice and a be a supplement element in $L$. If $L$ is amply generalized supplemented, then the quotient sublattice a/0 is amply generalized supplemented.

Proof. Clear.

Theorem 4. Let $L$ be a lattice. If $L$ is amply generalized supplemented, then for every $a \in L$, there exist $x, y \in L$ such that $x$ is a generalized supplement in $L$, $a=x \vee y$ and $y \leq r(L)$.

Proof. Let $b$ be a generalized supplement of $a$ in $L$ and $x$ be a generalized supplement of $b$ in $L$ with $x \leq a$. Since $b$ is a generalized supplement of $a$ in $L, a \vee b=1$ and $a \wedge b \leq r(b / 0)$. Since $x$ is a generalized supplement of $b$ in $L, x \vee b=1$ and $x \wedge b \leq r(x / 0)$. Let $y=a \wedge b$. This case $y=a \wedge b \leq r(b / 0) \leq r(L)$. Since $x \vee b=1$ and $x \leq a, a=a \wedge 1=a \wedge(x \vee b)=x \vee(a \wedge b)=x \vee y$.

Lemma 9. Let $L$ be a lattice and $a, b \in L$ such that $a \leq b$. Then the following assertions are equivalent.

(a) $b$ lies above $a$ in $L$.

(b) $a \vee x=1$ for every element $x \in L$ with $b \vee x=1$.

Proof. $(a) \Rightarrow(b)$ Let $b$ lies above $a$ in $L$. Then $b \ll 1 / a$. Let $b \vee x=1$ with $x \in L$. Then $b \vee a \vee x=1$, and since $a \vee x \in 1 / a$ and $b \ll 1 / a$, then $a \vee x=1$.

$(b) \Rightarrow(a)$ Let $b \vee x=1$ with $x \in 1 / a$. By hypothesis, $a \vee x=1$ and since $x \leq a$, $x=a \vee x=1$. Thus $b$ lies above $a$.

Lemma 10. Let $L$ be a lattice. If every element of $L$ lies above an element $x$ in $L$ such that $x / 0$ is generalized supplemented, then $L$ is amply generalized supplemented. 
Proof. Let $a \vee b=1$. By hypothesis, $a$ lies above an element $x$ in $L$ such that $x / 0$ is generalized supplemented. Since $a \ll 1 / x$, by Lemma $9, b \vee x=1$. Let $y$ be a generalized supplement of $b \wedge x$ in $x / 0$. This case $1=b \vee x=b \vee y$ and $b \wedge y \leq r(y / 0)$. Hence $y$ is a generalized supplement of $b$ in $L$ with $y \leq a$.

Corollary 3. Let $L$ be a lattice. If a/0 is generalized supplemented for every $a \in L$, then $L$ is amply generalized supplemented.

Proof. Clear from Lemma 10, because every element $a$ of $L$ lies above $a$ in $L$.

We can prove this Corollary directly as follows:

Let $a, b \in L$ with $a \vee b=1$. Since $a \wedge b \in b / 0$, by assumption, there is a generalized supplement $c$ of $a \wedge b$ in $b / 0$. That is, $c \in b / 0,(a \wedge b) \vee c=b$ and $a \wedge c=$ $a \wedge b \wedge c \leq r(c / 0)$. Since $a \vee b=1$ and $(a \wedge b) \vee c=b, 1=a \vee b=a \vee(a \wedge b) \vee c=$ $a \vee c$. Hence $c$ is a generalized supplement of $a$ in $L$ with $c \leq b$. Thus $L$ is amply generalized supplemented.

Example 1. Let $\Omega$ be family of all the submodules of $\mathbb{Z}$-module $\mathbb{Q}$. $\Omega$ is a lattice with $\subseteq$. This case, for $K, L \in \Omega, K \vee L=\sup \{K, L\}=K+L, K \wedge L=$ inf $\{K, L\}=K \cap L . \quad \Omega$ is generalized supplemented but not supplemented [7] . Since $\mathbb{Z}$ is a Dedekind domain, $\mathbb{Q}$ is a quotient field of $\mathbb{Z}$ and $\mathbb{Z}$ is not local, $\Omega$ is not amply generalized supplemented.

\section{REFERENCES}

[1] R. Alizade and S. E. Toksoy, "Cofinitely weak supplemented lattices," Indian Journal of Pure and Applied Mathematics, vol. 40, no. 5, pp. 337-346, 2009.

[2] R. Alizade and S. E. Toksoy, "Cofinitely supplemented modular lattices," Arabian Journal for Science and Engineering, vol. 39, pp. 919-923, 2011, doi: 10.1007/s13369-011-0095-z.

[3] G. Calugareanu, Lattice Concepts of Module Theory. Kluwer Academic Publishers, 2000.

[4] J. Clark, C. Lomp, N. Vanaja, and R. Wisbauer, Lifting Modules: Supplements and Projectivity in Module Theory. Basel: Frontiers in Mathematics, Birkhäuser, 2006. doi: 10.1007/3-7643-75736.

[5] Y. Wang and N. Ding, "Generalized supplemented modules," Taiwanese Journal of Mathematics, vol. 10, no. 6, pp. 1589-1601, 2006.

[6] R. Wisbauer, Foundations of Module and Ring Theory. Philadelphia: Gordon and Breach, 1991.

[7] H. Zöschinger, "Komplementierte moduln Über dedekindringen,” J. Algebra, vol. 29, pp. 43-56, 1974. 
Authors' addresses

Çiğdem Biçer

Ondokuz Mayıs University, Department of Mathematics, Kurupelit, Atakum Samsun, Turkey

E-mail address: cigdem_bicer184@hotmail.com

Celil Nebiyev

Ondokuz Mayıs University, Department of Mathematics, Kurupelit, Atakum Samsun, Turkey

E-mail address: cnebiyev@omu.edu.tr

Ali Pancar

Ondokuz Mayıs University, Department of Mathematics, Kurupelit, Atakum Samsun, Turkey

E-mail address: apancar@omu.edu.tr 